\title{
L'IMPACT DE L'HOMME SUR LES MILIEUX PALUSTRES : LES MARAIS D'ARLEUX
}

\author{
par E. VIGNEUX \\ Première Région Piscicole, 3, rue Sainte-Marie, 60200 COMPIEGNE
}

Au cours d'une étude demandée par la Fédération des A.P.P. du Nord, et destinée à proposer des aménagements piscicoles et halieutiques sur les marais d'ARLEUX (Nord), nous avons fait quelques observations sur l'impact de l'homme sur les milieux palustres.

Nous ne nous préoccuperons pas ici des problèmes de pollution urbaine, agricole, industrielle, qui sont d'ailleurs relativement peu importants au niveau de ces marais.

Notre attention s'est portée sur un phénomène en pleine évolution dans cette vallée: le tourisme. 
Le but de cet article est d'essayer de définir brièvement les incidences sur le milieu palustre des activités de loisir (pêche, chasse), et d'une conséquence indirecte de ces activites: la prolifération des bungalows et autres rés:dences secondaires au voisinage des plans d'eau.

\section{1. - LA PECHE}

\section{1. - La pêche en barque:}

Ce mode de pêche nécessite des plans d'eau assez vastes et relativement profonds (c'est le cas des marais d'ARLEUX, regroupant deux plans d'eau. I'un de 15 hectares, l'autre de 20 hectares, et de profondeur moyenne de $2,50 \mathrm{~m}$ ).

Dans ce cas, l'aménagement des berges est inutile et permet le développement des roselières, que le pêcheur en barque affectionne puisqu'elles constituent des cachettes pour le poisson et des abris contre le vent.

La pèche en barque parait donc compatible avec un certain développement de la végétation et cette activité ne semble pas entrainer de grandes modifications phytosociologiques: cependant, un problème se pose : où mettre la barque lorsque la journée de pêche est finie?

Sur les marais d'ARLEUX, les barques sont amarrées sur des emplacements loués par la commune. Ces emplacements s'étendent sur toute une berge avec destruction partielle des roselières.

On observe actuellement une augmentation du nombre de pêcheurs, c3 qui se traduit par une intensification de la circulation sur le marais; sa tranquillité se trouve donc perturbée, ce qui chasse les oiseaux (particulièrement les troupes de migrateurs).

D'autre part, cette augmentation du nombre de barques nécessite la création de nouvelles places d'amarrage, ce qui se traduit par le recul des roselières et la disparition d'autant de niches écologiques.

Malgré tout, l'impact de la pêche en barque sur le milieu naturel demeure assez faible.

\section{2. - La pêche du bord}

Nombreux sont les pêcheurs qui préfèrent la pêche du bord; en effet, posséder une barque est assez astreignant (entretien du bateau, location de l'emplacement).

Organiser la pêche du bord, c'est donc s'adresser à un plus grand public.

Mais c'est aussi modifier le milieu puisqu'il faut rendre les berges:

- facilement accessibles (création de routes ou de chemins, avec consolidation du sol au voisinage des marais, et débroussaillement des bordures);

- facilement pêchables (création de chenaux de pêcíe, avec desiruction de la végétation aquatique riveraine, représentant un abri et une zone de frayère pour le poisson). 
Ces transformations peuvent être plus ou moins importantes, et les schémas suivants (Fig. 1) présentent l'évolution possible d'un étang selon les aménagements.

\section{PECHE}

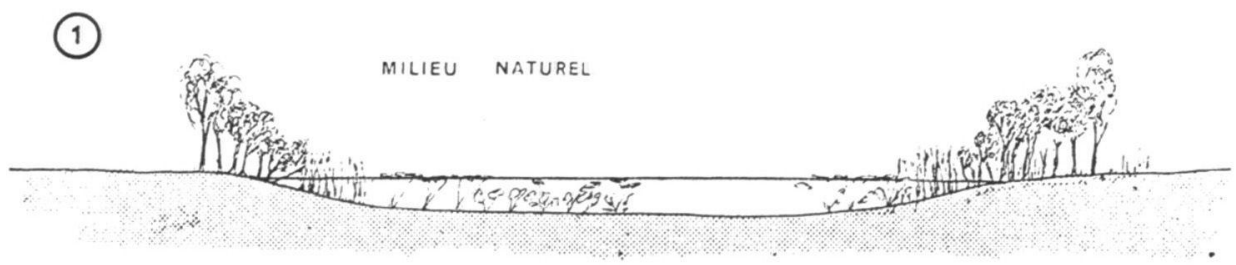

(2)

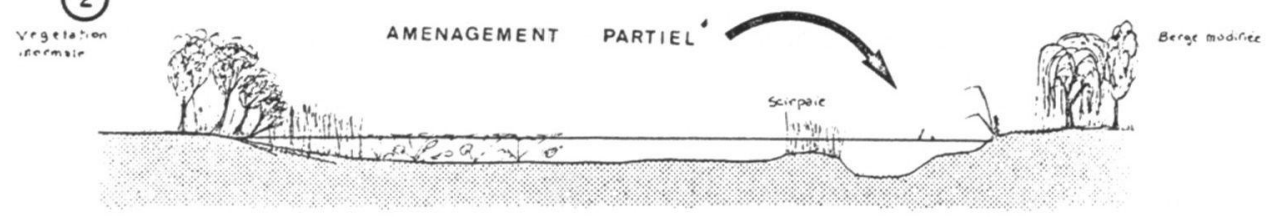

(3)

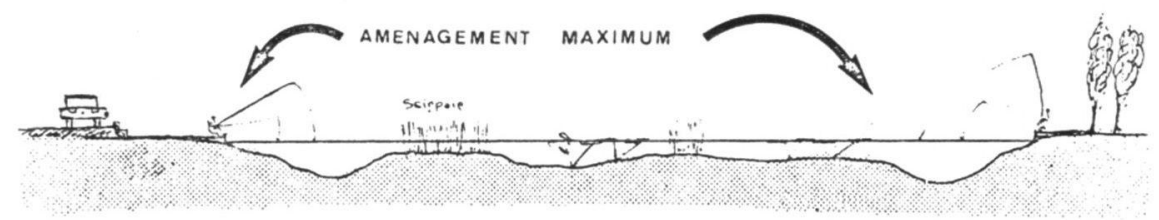

CHASSE

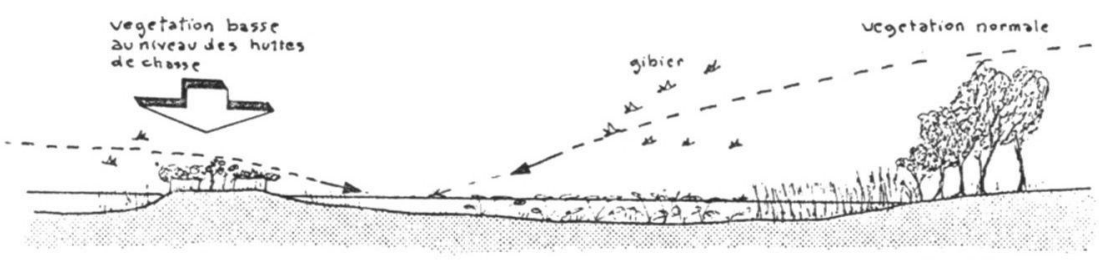

Fig. 1 - Schémas d'aménagements possibles d'un étang pour la pêche et pour la chasse 
TRANSECT No 1

Ce transect correspond à un milieu sauvage (compatible avec une pêche er barque modérée).

\section{TRANSECT No 2}

Nous observons sur ce schéma un aménagement partiel, limité à une berge; celle-ci présente une destruction de la végétation d'origine, notamment les saulaies à Salix viminalis et Salix triandra (qui poussent le pied dans l'eau, et empêchent l'accès aux rives), les mégaphorbiaies (végétation herbacée haute), les phragmitaies et cariçaies.

La scirpaie, poussant en eau plus profonde peut être préservée; elle cffre un abri aux poissons en lisière de la zone de pêche et peut protéger le chenal de pêche contre le vent

\section{TRANSECT $\mathbf{N}^{\circ} 3$}

Traduisant un aménagement intense, avec une importante transformation des berges, par:

- destruction de la végétation des rives et des frayères périphériques,

- création de chenaux de pêche, de berges soutenues, de digues, et même de routes le long des plans d'eau,

- diminution de la circonférence totale du plan d'eau par réalignement des berges, entrainant une diminution de la productivité totale. biotope.

On aboutit dans ce cas à un milieu très artificiel, et à la destruction du

Bien souvent, ce type d'aménagement conduit à une surexploitation, nécessitant des réempoissonnements fréquents. Ceci fait apparaitre un nouveau problème : celui de l'introduction dans un milieu d'espèces qui lui sont étrangères.

\section{2. - LA CHASSE}

Cette activité s'est développée parallèlement à la pêche. Sur la quarantaine d'hectares de plans d'eau que représentent les marais d'ARLEUX et leurs annexes, on ne compte pas moins de 15 huttes de chasse.

Outre la disparition du gibier, la chasse à la hutte semble avoir une incidence sur le milieu naturel:

- le chasseur a tendance à limiter l'extension des roselières pouvant constituer des cachettes pour le gibier;

- de plus, il sélectionne une végétation arbustive pour camoufler sa hutte, les grands arbres coupant le vol des oiseaux.

De telle sorte que l'on observe un faciès assez caractéristique au voisinage des huttes, le chasseur modifiant le milieu pour se rapprocher de la situation idéale (cf. Fig. 1). 


\section{3. - PROBLEMES TOURISTIQUES}

Dans cette vallée ayant conservé son caractère sauvage, bien qu'étant proche des grands centres industriels du Douaisis et de la région Lilloise, l'activité touristique s'est considérablement développée.

Parallèlement aux activités de loisir liées aux plans d'eau (chasse, pêche, canotage), on observe une véritable prolifération de bungalows et de résidences secondaires au voisinage des marais.

Cette situation conduit bien souvent à une double appropriation des berges:

- par les propriétaires des bungalows qui limitent ou ferment les accès aux plans d'eau.

- par les propriétaires de barques, dont les amarrages s'étendent souvent tout au long des rives.

Ces bungalows se sont implantés de manière anarchique, sur du terrain loué aux communes, sans permis de construire et la plupart du temps sans assainissement.

Ces zones sont évidemment surpeuplées en période estivale, surtout pendant les week-ends.

Mais, outre le préjudice esthétique et écologique causé par ces bungalows, nous n'avons pas observé d'influence nette sur la qualité des eaux dans le cas précis des marais d'ARLEUX.

Ce faible impact sur la qualité des eaux, malgré l'absence d'assainissement, semble s'expliquer essentiellement par le fait que les bungalows ne sont pas reliés au réseau d'adduction d'eau.

Le volume des rejets est peu important; ceux-ci sont diffus et en partie épurés dans le sol avant d'atteindre le plan d'eau.

\section{UN CERTAIN NOMBRE DE REMARQUES S'IMPOSENT}

Il est souvent difficile de doser les aménagements destinés à développer et rationaliser une activité de loisir. Chaque cas est en effet un cas d'exception, qu'il convient d'étudier avec attention afin d'éviter des erreurs lourdes de conséquences pour le milieu.

Une étude préliminaire du biotope est donc indispensable afin d'adapter l'aménagement.

C'est ainsi que, pendant un an, notre attention s'est portée sur les marais d'ARLEUX (étude de la flore et de la faune microscopique et macroscopique, de la qualité chimique et bactériologique des eaux, de la topographie, de la climatologie...).

\section{En conclusion :}

Le milieu que nous avons étudiè commence à souffrir de l'intensification de la fréquentation humaine. 
II nous est apparu utile de définir une zone de protection où l'influence humaine ne risque pas de perturber le développement de la faune et de la flore. qui sont très diversifiés dans les zones encore intactes.

C'est dans cet esprit que les aménagements proposés pour les marais d'ARLEUX présentent deux aspects:

- des mesures de protection,

- des mesures destinées à favoriser la pratique de la pêche.

\section{1. - Les mesures de protection}

- la circulation en barque sur l'un des marais est exclusivement réservée aux pêcheurs,

- certaines zones sont fermées au public,

- par suite d'un accord entre la commune d'ARLEUX et la Fédération des A.P.P. du Nord, les nouvelles constructions de bungalows sont interdites en bordure de marais, et les amarrages de barque sont strictement interdits sur l'un des deux marais.

\section{2. - Les mesures favorisant la pratique de la pêche} (mesures proposées à la Fédération des A.P.P. du Nord) petit port.

- Libération d'une berge encombrée par les barques par la création d'un libérée.

- Aménagement pour la pêche à la ligne d'une portion de la rive ainsi

La priorité est ainsi laissée à la pêche en barque, celle qui présente l'impact le plus faible sur le milieu naturel et pour laquelle les marais d'ARLEUX semblent le mieux adaptés (plans d'eau relativement vastes et profonds, roselières situées en bordure).

\section{BIBLIOGRAPHIE}

DEWAILLY J.-M. - Utilisation des sols et aménagement de la vallée de la Sensée - 1966.

DIRECTION DEPARTEMENTALE DE L'EQUIPEMENT DU NORD, GEP DE LILLE ET DE DOUAI - Etude de l'évolution des sites et du paysage de la Sensée pour la préparation d'un schéma de secteur - 1972.

MERIAUX J.-L. - Etude de la végétation de la mare à Goriaux.

Parce régional National de Saint-Amant - Raismes

DEA de Biologie Végétale - Université de LILLE I (mai 1974).

SRAE NORD - PAS-DE-CALAIS - Etude de la qualité des eaux de la Sensée Document provisoire - Septembre 1975.

VIGNEUX-QUENTIN D. et VIGNEUX E. Les marais d'Arleux - Proposition d'aménagement - Novembre 1975. 